\title{
Repeated soil applications of fungicide reduce activity against cavity spot in carrots
}

\author{
James J. Farrar J. Joseph Nunez $\square$ R. Michael Davis
}

In recent years, carrot growers in the San Joaquin Valley have suffered economic losses due to cavity spot, a soilborne disease, despite frequent applications of the fungicide mefenoxam. Although the pathogen remained highly sensitive to mefenoxam in laboratory studies, the effective dosage of the fungicide was apparently compromised in certain fields. Compared to its longevity in soils with no history of mefenoxam use, such as fields using organic production methods, the fungicide degraded rapidly in soil from fields with repeated mefenoxam use. Our research reveals that repeated applications of the fungicide to soil can increase the activity of microorganisms that degrade it, potentially reducing its efficacy against cavity spot. This is problematic in California since mefenoxam is the only fungicide available to carrot growers for cavity spot control. It may be prudent to practice long crop rotations and to limit use of mefenoxam, where possible.

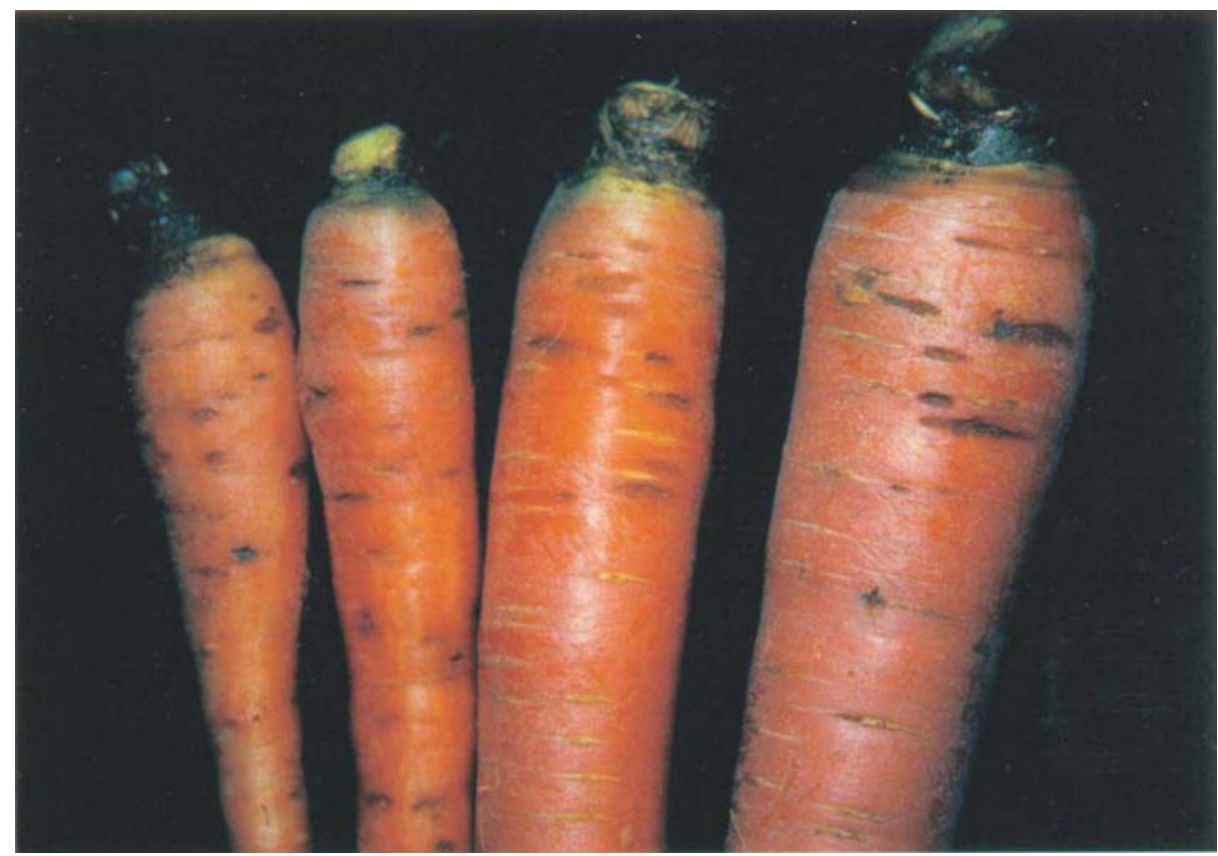

Carrots infected with cavity spot are usually deemed unsuitable for the fresh market. Caused by the fungus Pythium, cavity spot can also infect other crops such as beets, broccoli and wheat.

Cavi avity spot is one of the most damaging diseases of carrots in the San Joaquin Valley, where more than 50,000 acres are planted annually. Although the disease does not reduce carrot weights, infected carrots are deemed unsuitable for the fresh market due to the unsightly blemishes. The economic impact of the disease can be substantial. In extreme cases, growers have abandoned fields of carrots with high levels of cavity spot.

The disease is characterized by root lesions that are elliptical (oriented across the breadth of the root), generally less than 0.5 inch in length, and shallow. The lesions are generally more numerous on the upper third of the taproot. There are no symptoms on the aboveground parts of the plant.
Cavity spot is caused by at least two species of Pythium, including $P$. violae and $P$. ultimum. In California, cavity spot is most often attributed to $P$. violae (Vivoda et al. 1991). Little is known about the life cycle and population dynamics of $P$. violae in soil. It is assumed that the fungus survives as thick-walled oospores, which germinate in response to sugars and other nutrients that exude from roots. Because the fungus has a wide host range on such diverse plants as alfalfa, beets, blackeye beans, broccoli, celery, cucumber and wheat, it probably persists in the soil for years (Schrandt et al. 1994). None of the carrot cultivars commonly grown in California possess useful levels of resistance. Growers have observed that disease 
incidence in a field increases in proportion to the number of successive carrot crops.

Timely harvests and good water management can help reduce the occurrence of cavity spot. Unfortunately, there is no accurate means of predicting disease risk in an individual field, and applications of a fungicide are sometimes necessary. Today, only one fungicide, mefenoxam (Ridomil Gold $\mathrm{EC}$ ), is registered to control cavity spot on carrots. Applied to the soil up to three times in a growing season, it usually results in adequate disease control (Davis et al. 1991). Recently, however, the fungicide has failed to control the disease in a number of fields. In at least two fields, the entire carrot crop was abandoned due to a high incidence of cavity spot. The purpose of this study was to determine the cause of the reduced efficacy of mefenoxam on carrots in the San Joaquin Valley.

\section{Isolate sensitivity}

We first examined the possibility that the loss of cavity spot control was due to the development of resistance to mefenoxam within the pathogen population. To obtain isolates of the pathogen, carrots with cavity spot lesions were collected from 13 fields from 1998 to 2000 . The history of mefenoxam applications ranged from no applications in organic fields to many applications in conventional fields, including those fields where mefenoxam failed to control cavity spot. After the carrots were washed in running tap water, cavity spot lesions and a minimal amount of surrounding healthy tissue were removed with a sterilized scalpel and plated on corn- meal agar amended with antibiotics. When the colonies were 2 to 4 days old, the isolates were transferred to and subsequently maintained on cornmeal agar plates. Isolates were identified based on morphological characteristics of the colonies grown on sterile grass blades in water culture. Pathogenicity of the isolates was confirmed by placing 4-millimeterdiameter agar plugs from the margin of 3-day-old cultures on surfacedisinfested carrots. After the sites of inoculations were covered with. sterile moist cotton, the carrots were incubated for 14 days at $68^{\circ} \mathrm{F}\left(20^{\circ} \mathrm{C}\right)$ in the dark.

A total of 31 isolates of $P$. violae and 26 isolates of $P$. ultimum pathogenic to carrot were evaluated for sensitivity to mefenoxam. Four-millimeter-diameter plugs from the margin of 3-day-old cultures of each isolate were placed on cornmeal agar plates amended with 0 , $0.1,1,10,50$ or 100 parts per million (ppm) mefenoxam. Colony diameters were measured after the plates were incubated at room temperature in the dark for 3 days. Each treatment was replicated three times.

Based on the inhibition of the growth of the colonies on mefenoxamamended agar, all $P$. violae and $P$. ultimum isolates were sensitive to the fungicide (fig. 1). The average colony diameter of both species on plates amended with 1 ppm mefenoxam was less than $40 \%$ of the colony diameter on unamended agar. These results were similar to the results of our unpublished 10-year study on the sensitivity of Pythium spp. to metalaxyl and mefenoxam, which is the active isomer of metalaxyl, and the only compound in use today on carrots. To date, no re-

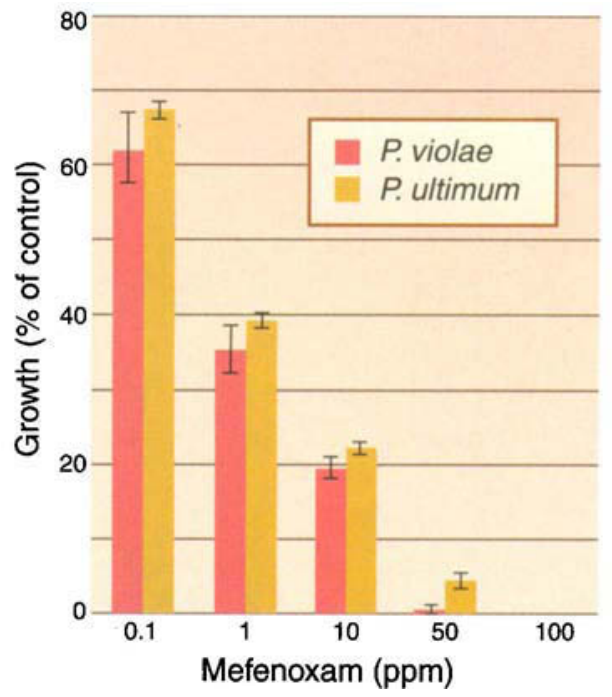

Fig. 1. Relative growth of Pythium violae (31 isolates) and $P$. ultimum (26 isolates) on cornmeal agar amended with various concentrations of the fungicide mefenoxam. Colony diameters were expressed as a percentage of the colony diameter on unamended cornmeal agar. Bars represent standard errors. Both fungi were highly sensitive to the fungicide.

sistance to metalaxyl or mefenoxam has been identified in carrot isolates of $P$. violae and $P$. ultimum.

\section{Degradation of mefenoxam}

Since we found no evidence that isolates of $P$. violae and P. ultimum were insensitive to mefenoxam, we designed two experiments to determine whether mefenoxam degraded more rapidly in soil where it had been used repeatedly. An increased rate of metalaxyl (and, therefore, mefenoxam) degradation has developed in avocado, citrus and tobacco soils with a history of metalaxyl use (Droby and Coffey 1991).

Soil was collected from four conventionally farmed carrot fields in the San Joaquin Valley with a history of repeated mefenoxam applications, and from four certified organic fields. Each field served as a replication (the trial was repeated once with a different set of fields). Soil from the surface to 8 


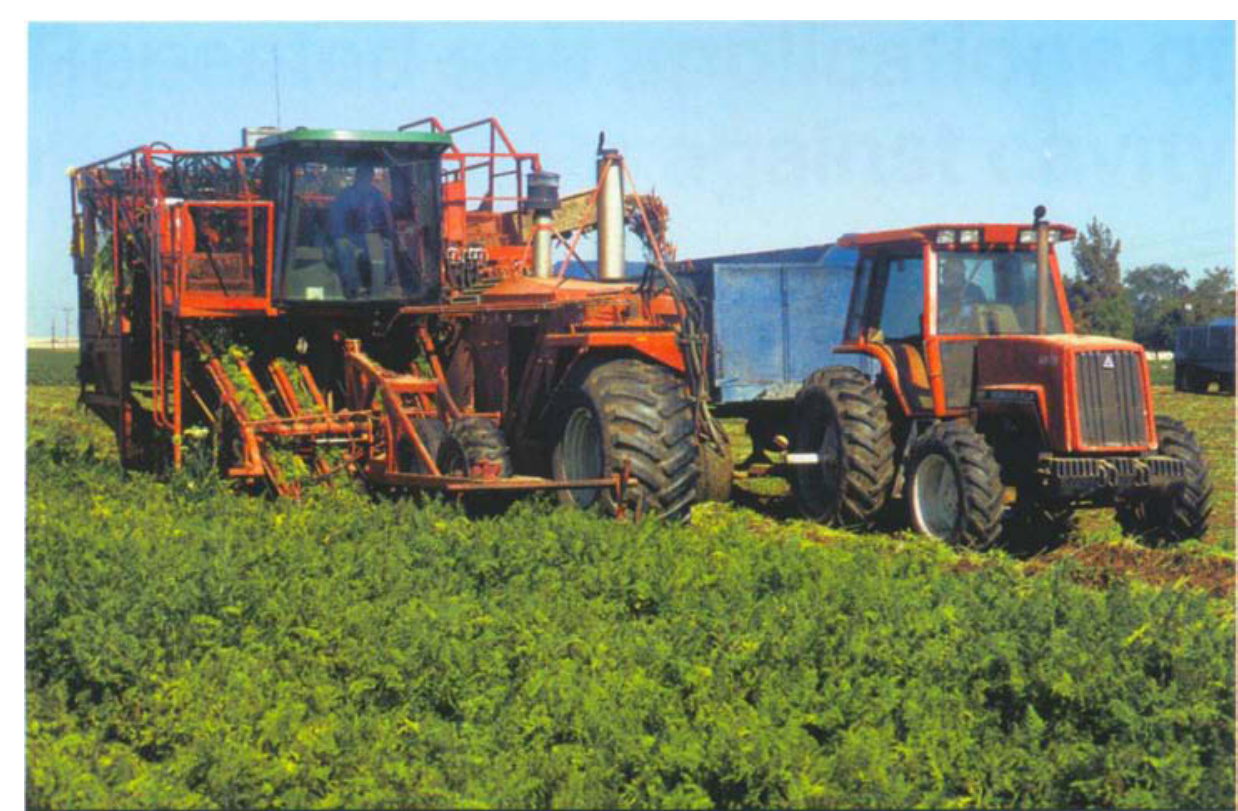

The fungicide mefenoxam initially provides effective control of cavity spot, but after repeated applications over several years its efficacy can be reduced. Carrots are harvested in Kern County.

inches deep was collected in a $\mathrm{W}-$ shaped pattern across each field with a soil core that was 1 inch in diameter. Twenty cores from each field were bulked, sifted and placed in a large plastic tub to receive and facilitate the incorporation of 350 microliters $(\mu 1)$ mefenoxam in 50 milliliters deionized water. In order to quantify the degradation of the fungicide, the amount added to the soil was 100 times the labeled rate. Immediately after the fungicide was thoroughly mixed into the soil, a 100-cubic-centimeter (cc) sample of soil was collected and dried in a $158^{\circ} \mathrm{F}\left(70^{\circ} \mathrm{C}\right)$ oven. Once dried, the soil was ground with a mortar and pestle and stored in a plastic bag. This process was repeated at 4-day intervals for 16 days. Between samplings, the soil was stored at room temperature in a plastic pot with a top diameter and depth of 5 inches. The soil moisture content was adjusted to approximately field capacity by the addition of deionized water each time the soil was sampled. A 200-cubic-centimeter aliquot (portion) of soil collected prior to spiking with mefenoxam served as the control for each field.

\section{Bioassay designed, conducted}

In the first experiment on mefenoxam degradation, a bioassay designed to quantify mefenoxam activity was conducted with an isolate of $P$. violae (isolate ctl-4a) originally isolated in carrots from Kern County. The bioassay method was similar to methods used in earlier studies (Bailey and Coffey 1984; Wicks 1988). An aliquot of 50 grams of dried soil from each sample was agitated in 100 milliliters deionized water for 1 hour on an orbital shaker at $150 \mathrm{rpm}$. After the soil suspension was filtered through \#1 Whatman paper, cornmeal agar was added to the filtrate at a rate of 0.017 grams of cornmeal agar per milliliter of filtrate. The amended agar was then autoclaved and poured into three petri plates. A 4-millimeter plug from the margin of a 3-day-old colony of each plate. After 4 days at room temperature, the colony diameter on each plate was measured. The experiment was performed twice and the data combined. The inhibition of colony growth on the fungicide-amended $P$. violae was placed in the center of

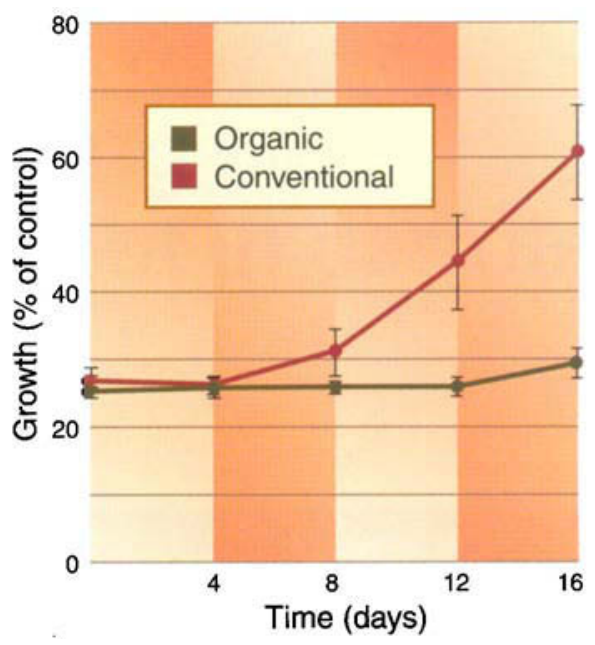

Fig. 2. Relative growth of Pythium violae on agar amended with the fungicide mefenoxam and soil extracts from four organic and four conventional fields. Bars represent standard errors. After 8 days, the fungicide in soil extracts from conventional fields was significantly less inhibitory to $P$. violae than in soil extracts from organic fields.

agar was expressed as a percentage of the diameter of colonies grown on plates amended with nonspiked soil. Data from the two trials were combined.

The colony diameter of $P$. violae on plates prepared from spiked organic soils over the duration of the experiment remained at $25 \%$ to $30 \%$ of the control (fig. 2). The mefenoxam applied to the organic soil on day zero was not markedly degraded during the 16 days of the experiment. In contrast, the colony diameter on plates prepared from conventionally farmed soils increased on days 12 and 16, indicating reduced activity of mefenoxam in these soils after 8 days.

\section{Soil analysis}

In the second experiment on mefenoxam degradation, a direct measurement of mefenoxam levels in soil was determined with a gas chromatograph (Farrar and Davis 2000). An aliquot of 5 grams of dried soil from each sample was added to 5 milliliters of methanol and shaken. After the methanol was allowed to evaporate at room temperature, the contents of the 


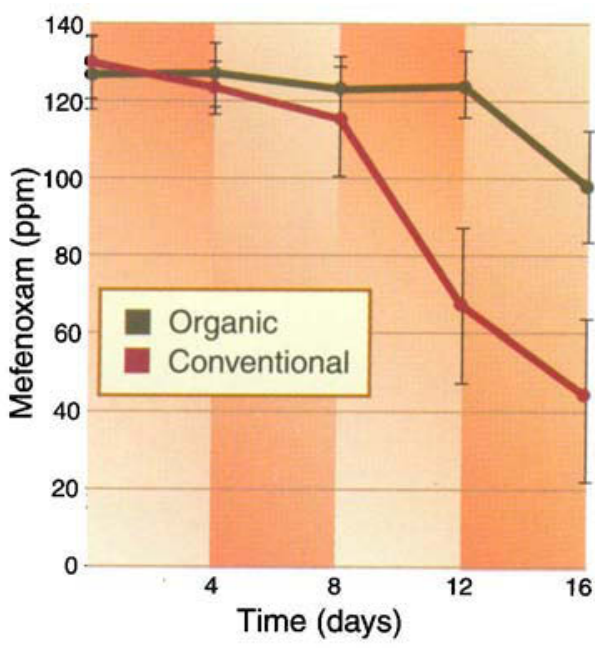

Fig. 3. Degradation of mefenoxam in four organic and four conventional field soils spiked with the fungicide. Mefenoxam concentration (ppm) was determined by gas chromatography. Bars represent standard errors. After 8 days, the concentration of mefenoxam in soils from conventional fields was significantly lower than that in organic soils.

vials were resuspended in 1 milliliter of hexane. Standards, controls and samples were analyzed in a gas chromatograph. The concentration of mefenoxam for each soil extraction was calculated using a standard curve prepared for each field. Data from the two trials were combined.

In the organic soil, the concentration of mefenoxam remained at about $125 \mathrm{ppm}$ from day zero to day 12 , and then decreased to $100 \mathrm{ppm}$ (fig. 3). In contrast, the concentration of mefenoxam in the conventional soil degraded rapidly after 8 days. At 16 days, the concentration of mefenoxam in soil from conventionally farmed fields was less than half the concentration of mefenoxam in the organic soils.

\section{Microflora degrades fungicide}

Both the bioassay and gas chromatography methods of quantifying the concentration of mefenoxam gave similar results. In the organic soils, mefenoxam levels decreased slightly over the 16 days of the experiment, whereas levels of mefenoxam in the

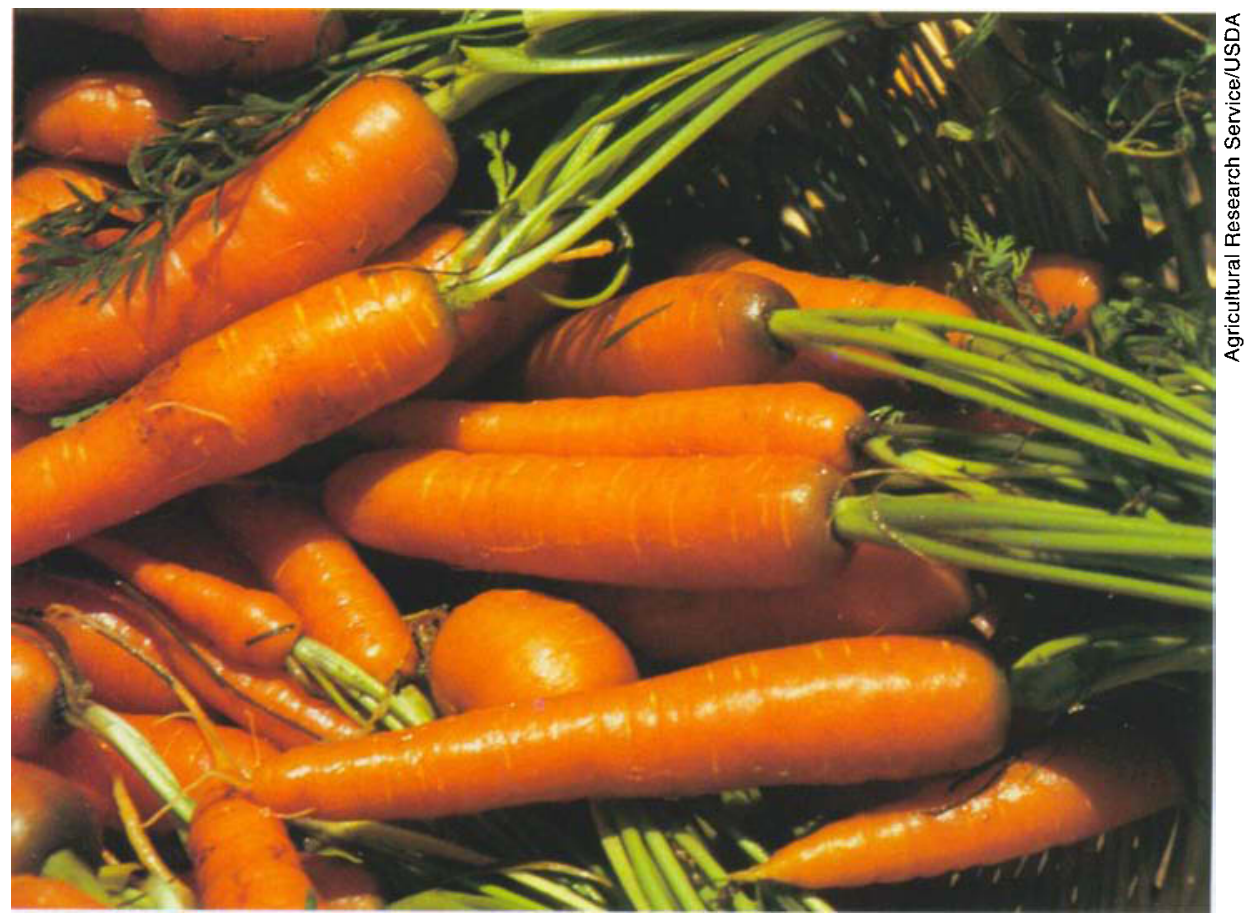

Soil microflora apparently degrades mefenoxam more rapidly after repeated applications. Carrot growers may need to rely on longer crop rotations and limit use of the fungicide in order to ensure a healthy crop.

conventional soils decreased rapidly after 8 days. These results indicate a more rapid degradation of mefenoxam in carrot field soils with a history of mefenoxam application.

Apparently, the soil microflora that degrades mefenoxam is stimulated after repeated applications of the fungicide (Bailey and Coffey 1985, 1986); they are stimulated simply because the fungicide is a source of nutrition.

This is problematic in California's carrot fields since mefenoxam is the only fungicide available to growers for cavity spot control. Because of the recent failures to control the disease, it may be prudent to practice long crop rotations and to limit the use of the fungicide, where possible.

I.J. Farrar is Assistant Professor, Plant Sciences Department, California State University, Fresno; J.J. Nunez is Farm Advisor, UC Cooperative Extension (UCCE), Kern County; and R.M. Davis is UCCE Specialist, Department of Plant Pathology, UC Davis.

\section{References}

Bailey AM, Coffey MD. 1984. A sensitive bioassay for quantification of metalaxyl in soils. Phytopath 74:667-9.

Bailey AM, Coffey MD. 1985. Biodegradation of metalaxyl in avocado soils. Phytopath 75:135-7.

Bailey AM, Coffey MD. 1986. Characterization of microorganisms involved in accelerated biodegradation of metalaxyl and metalachlor in soils. Can J Microbiol 32:562-9.

Davis RM, Nunez JJ, Guerard JP, Vivoda E. 1991. If registered, fungicide could reduce cavity spot in carrots. Cal Ag 45(2):29-30

Droby S, Coffey MD. 1991. Biodegradation process and the nature of metabolism of metalaxyl in soil. Ann Appl Biol 118:543-53.

Farrar JJ, Davis AM. 2000. Detection and quantification of metalaxyl using the MIDI Microbial Identification System. Phytopath 91:S184.

Schrandt JK, Davis RM, Nunez JJ. 1994. Host range and influence of nutrition, temperature and $\mathrm{pH}$ on growth of Pythium violae from carrot. Plant Dis 78:335-8.

Vivoda E, Davis RM, Nunez JJ, Guerard JP. 1991. Factors affecting the development of cavity spot on carrot. Plant Dis 75:519-22.

Wicks TJ. 1988. Effect of metalaxyl on the control of Phytophthora crown rot of almonds. Aust J Exp Agric 28:547-52. 